\title{
Acknowledgment of Ad Hoc Reviewers (2020)
}

The Editor would like to thank the following persons who served as reviewers for Psychology, Public Policy, and Law (PPPL), from September 2019 through August 2020.

Paul S. Appelbaum

Sydney Baker

Connie J. A. Beck

Arlin Benjamin

Craig Bennell

William Bernet

Peter Blanck

Uri Blasbalg

Karen Bogenschneider

Jeffrey P. Braden

Chuck Brainerd

Eve M. Brank

Sonja Brubacher

Maggie Bruck

Ray Bull

Judith Cashmore

Caitlin Cavanagh

Steve Charman

Hayley M. D. Cleary

Carl B. Clements

Simon A. Cole

Lois Condie

Tarika Daftary-Kapur

Milfred Bud Dale

Ann M. Delpha

Corine de Ruiter

Jason Dickinson

Eric Drogin

James Dunlea

Joel Dvoskin

Jennifer Dysart

Mitchell Eisen

James W. Ellis

Peter English

Steven K. Erickson

Jacqueline R. Evans

Jeffrey A. Fagan

Weyam Fahmy
Neal Feigenson

Ryan J. Fitzgerald

Mark Fondacaro

Karen Franklin

Lilli Friedland

Brandon Garrett

Loraine Ruth Renate Gelsthorpe

Paul Gill

Susan G. Goldberg

Gail S. Goodman

Jonathan W. Gould

William N. Gowensmith

Gisli Gudjonsson

Michelle Guyton

Angelica Veronica Hagsand

Emily Haney-Caron

Stephen D. Hart

Alan Hawkins

Johanna Hellgren

L. Maaike Helmus

Matthew Hiller

Melissa Hines

George W. Holden

Robert J. Homant

Ruth Horry

Matthew T. Huss

Jonathan Jackson

Saul Kassin

Christopher E. Kelly

Lauren E. Kois

Ivan Kruh

Jeff Kukucka

Jennifer Lavoie

Michael R. Leippe

Richard Leo

Terry M. Libkuman

Scott O. Lilienfeld
Kirk Luther

Mona Lynch

Phillip Magaletta

John M. Majer

Jamal K. Mansour

Kureva Matuku

Patrick McClanahan

Laura A. McCloskey

Troy McEwen

Richard McNally

Madelyn Simring Milchman

Quincy Miller

Michael H. Miner

John Monahan

Katrin Mueller-Johnson

Edward P. Mulvey

Wendy Murphy

Daniel C. Murrie

John E. B. Myers

Sherry Nakhaeizadeh

Nancy Ryba Panza

Kimberly A. Pavlik

Steven Penrod

Christina Perez

Michael Perlin

Bernard Perlmutter

Martine B. Powell

Jodi Quas

Richard Redding

Valerie Reyna

Kim P. Roberts

Mary R. Rose

Barry Ruback

Tiffany Russell

Michael Saks

Thomas Schacht

Kyle C. Scherr
Nick Schweitzer

Nicholas Scurich

Michael Seto

Alissa Sherry

Dan Simon

Diane Sivasubramaniam

Emma Sleath

Laura Smalarz

Andrew Smith

Steven M. Smith

Brent Snook

Jessica Snowden

Loretta Stalans

Margaret C. Stevenson

Veronica Stinson

Stacia N. Stolzenberg

Daniel O. Taube

Rick Trinkner

Gabby Trupp

Brent Turvey

Tom Tyler

Jonathan P. Vallano

Tinneke Van Camp

Jodi Viljoen

Prue Vines

Sara Waring

Richard A. Warshak

Nesa Wasarhaley

Lynn Weiher

Miko M. Wilford

Cynthia Willis-Esqueda

Barbara B. Woodhouse

Yueran Yang

Patricia A. Zapf

Tina M. Zottoli 